Acta Crystallographica Section E

Structure Reports

Online

ISSN 1600-5368

\section{Kassim F. Adebambo, Nicola M. Howarth and Georgina M. Rosair*}

Chemistry, William Perkin Building, School of Engineering \& Physical Sciences, Heriot-Watt University, Riccarton, Edinburgh EH14 4AS, Scotland

Correspondence e-mail: g.m.rosair@hw.ac.uk

\section{Key indicators}

Single-crystal X-ray study

$T=100 \mathrm{~K}$

Mean $\sigma(\mathrm{C}-\mathrm{C})=0.002 \AA$

$R$ factor $=0.034$

$w R$ factor $=0.095$

Data-to-parameter ratio $=14.8$

For details of how these key indicators were automatically derived from the article, see http://journals.iucr.org/e.

\title{
Benzyl 2-amino-6-chloro-9H-purine-9-carboxylate
}

The title compound, $\mathrm{C}_{13} \mathrm{H}_{10} \mathrm{ClN}_{5} \mathrm{O}_{2}$, crystallizes with two molecules in the asymmetric unit. These are connected by five hydrogen bonds, viz. three $\mathrm{N}-\mathrm{H} \cdots \mathrm{N}$ interactions, two longer $\mathrm{C}=\mathrm{O} \cdots \mathrm{H}-\mathrm{N}$ interactions, bifurcated at the $\mathrm{O}$ atom, and a $\mathrm{C}-\mathrm{H} \cdots \mathrm{N}$ contact.

\section{Comment}

The chemistry of purines has been largely driven in recent years by the desire to synthesize oligonucleotides and their analogues as well as novel purine-containing nucleosides for a wide range of medicinal applications (Vyle \& Howarth, 2001). We have previously reported the synthesis and polymerization of lipophilic polyamide nucleic acids (PNA) as potential colorimetric diagnostics (Howarth, Lindsell et al., 2003), and the design and synthesis of true peptide mimics of DNA for possible use as antigene agents (Howarth \& Wakelin, 1997; Howarth, Wakelin \& Walker, 2003). During these studies, we have encountered numerous difficulties in preparing the required $\mathrm{N}$-2-benzyloxycarbonyl-protected guanine monomers from 2-amino-6-chloropurine (Howarth \& Wakelin, 1997). Inspired by the work reported by Dey \& Garner (2000) on the synthesis of tris-tert-butoxycarbonyl 2-amino-6chloropurine, we decided to employ a similar strategy for preparing these monomers. As had been found by Dey \& Garner (2000), this reaction afforded a single product. However, analysis of the product by ${ }^{1} \mathrm{H}$ NMR spectroscopy showed the presence of only one benzyloxycarbonyl group rather than three, which had been the case when 2-amino-6chloropurine was treated with di-tert-butyl dicarbonate under analogous conditions (Dey \& Garner, 2000). The exact identity of the monobenzyloxycarbonyl-protected product was revealed to be that of the title compound, (I), by a singlecrystal X-ray study.<smiles>Nc1nc(Cl)c2ncn(C(=O)OCc3ccccc3)c2n1</smiles>

(I)
Received 8 December 2004 Accepted 19 January 2005 Online 29 January 2005
C 2005 International Union of Crystallography Printed in Great Britain - all rights reserved
Compound (I) crystallizes as two crystallographically independent molecules ( $A$ and $B$ ) (Fig. 1). These differ in the relative ring orientations about the $\mathrm{C} 10-\mathrm{N} 9$ bonds $[\mathrm{C} 4 A-$ $\mathrm{N} 9 A-\mathrm{C} 10 A-\mathrm{O} 10 A=-5.0(2)^{\circ}$ and $\mathrm{C} 4 B-\mathrm{N} 9 B-\mathrm{C} 10 B-$ 


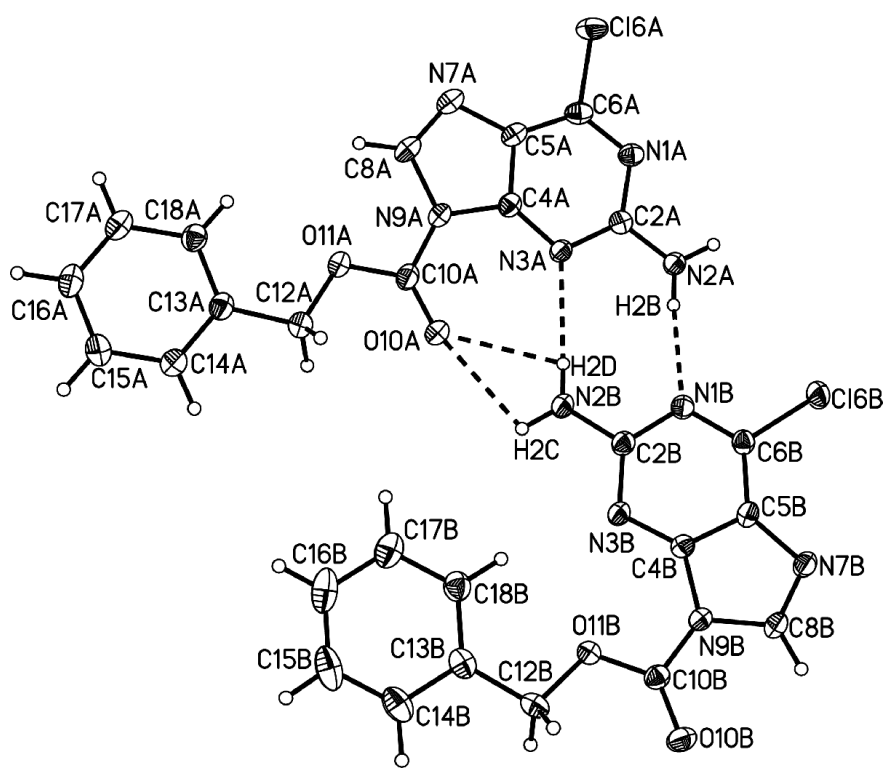

Figure 1

Perspective view of the asymmetric unit in (I), with hydrogen bonds shown as dashed lines. Displacement ellipsoids are shown at the $50 \%$ probability level and $\mathrm{H}$ atoms have arbitrary radii of $0.1 \AA$ for clarity.

$\left.\mathrm{O} 10 B=-173.60(13)^{\circ}\right]$. The independent molecules $A$ and $B$ have different hydrogen-bonding arrangements. There is extensive hydrogen bonding between the two crystallographically independent molecules. They are connected by five intermolecular hydrogen bonds $[\mathrm{N} 2 A-\mathrm{H} 2 B \cdots \mathrm{N} 1 B$, $\mathrm{N} 2 B-\mathrm{H} 2 D \cdots \mathrm{N} 3 A, \quad \mathrm{~N} 2 B-\mathrm{H} 2 C \cdots \mathrm{O} 10 A, \quad \mathrm{~N} 2 B-\mathrm{H} 2 D \cdots$ $\mathrm{O} 10 A$ and $\mathrm{N} 2 A-\mathrm{H} 2 B \cdots \mathrm{N} 7 B^{\mathrm{i}}$ [symmetry code: (i) $2-x, y-$ $\frac{1}{2}, \frac{1}{2}-z$; Table 1], where the $\mathrm{N}-\mathrm{H} \cdots \mathrm{N}$ contacts are the shortest. The first four hydrogen bonds are shown in Fig. 1. The hydrogen-bonding links between molecules $A$ and $B$ result in the formation of two eight-membered rings. The $\mathrm{N}-$ $\mathrm{H} \cdot \mathrm{N}$ contacts have a symmetrical carboxylic acid dimer motif, $R_{2}^{2}(8)$ (Bernstein et al., 1995). The geometry of the $\mathrm{N}-$ $\mathrm{H}$. . O contact is very different, the angles at $\mathrm{H} 2 \mathrm{C}$ and $\mathrm{H} 2 \mathrm{D}$ being $101.6(13)$ and $101.1(13)^{\circ}$, respectively. The fifth intermolecular contact is another $\mathrm{N}-\mathrm{H} \cdots \mathrm{N}$ contact, $\mathrm{N} 2 A-$ $\mathrm{H} 2 B \cdots \mathrm{N} 7 B^{\mathrm{i}}$, which is almost parallel to the $c$ axis and gives rise to an infinite chain that runs parallel to the $b$ axis, shown in Fig. 2. However, N7 $A$ does not take part in such a close intermolecular contact. The closest contact for $\mathrm{N} 7 A$ is $\mathrm{C} 8 B-$ $\mathrm{H} 8 B \cdots \mathrm{N} 7 A^{\mathrm{ii}}$ [symmetry code (ii) $\left.1+x, 1+y, z\right]$.

\section{Experimental}

Dibenzyl dicarbonate $(2.40 \mathrm{ml}, 9.42 \mathrm{mmol}, 4$ equivalents $)$ was added to a stirred solution of 2-amino-6-chloropurine $(0.40 \mathrm{~g}, 2.36 \mathrm{mmol}, 1$ equivalent) and DMAP (dimethylaminopyridine, $0.03 \mathrm{~g}, 0.1$ equivalent) in anhydrous dimethylformamide $(50 \mathrm{ml})$ at room temperature under argon, and the resulting mixture was left to stir for $18 \mathrm{~h}$. Subsequently, the solvent was removed in vacuo and the residue was purified by column chromatography using ethyl acetate/petroleum ether (2:1) as the eluting solvent. The product-containing fractions

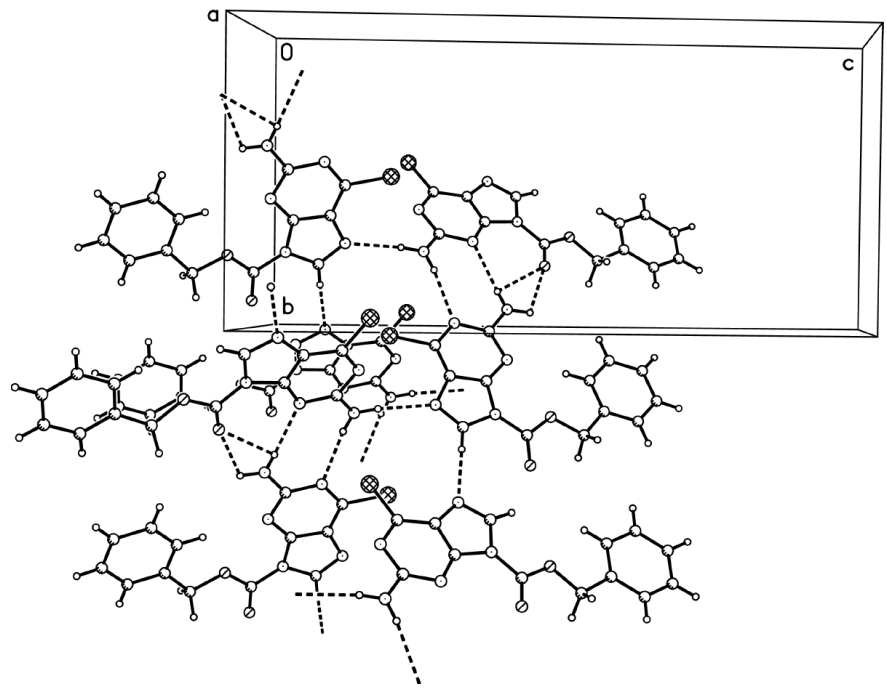

Figure 2

View of the packing arrangement for (I). Dashed lines indicate hydrogen bonds.

were combined to afford a brown oily solid, which was further purified by trituration with diethyl ether to give (I) as a colourless solid (yield $0.80 \mathrm{~g}, 26 \%$ ). Compound (I) was crystallized from deuterochloroform. M.p. 417-418 K; $R_{\mathrm{f}} 0.35$ (ethyl acetate/petroleum ether, 2:1). Analysis found: C 51.18, H 3.32, N 22.95\%; $\mathrm{C}_{13} \mathrm{H}_{10} \mathrm{O}_{2} \mathrm{~N}_{5} \mathrm{Cl}$ requires: C 51.41, H 3.32, N 23.06\%. v $\max \left(\mathrm{KBr}, \mathrm{cm}^{-1}\right)$ : 3497, 3313, 3198, 1775, 1742, 1626, 1561, 1512, 1485, 1395, 1368, 1301, 1192, 1175 and 1107; ${ }^{1} \mathrm{H}$ NMR $\left(200 \mathrm{MHz}, \mathrm{CDCl}_{3}\right): \delta 5.49(s, 2 \mathrm{H}), 5.64($ br s, $2 \mathrm{H})$, $7.34-7.53(m, 5 \mathrm{H}), 8.23(s, 1 \mathrm{H}) ;{ }^{13} \mathrm{C}$ NMR $\left(50 \mathrm{MHz}, \mathrm{CDCl}_{3}\right): \delta 70.2$, 128.8, 129.2, 133.5, 139.6, 147.2, 152.4, 153.0, 160.4. NMR spectra were recorded on Bruker DPX400 and AC200 spectrometers, from $\mathrm{CDCl}_{3}$ solutions at $293 \mathrm{~K}$.

\section{Crystal data \\ $\mathrm{C}_{13} \mathrm{H}_{10} \mathrm{ClN}_{5} \mathrm{O}_{2}$ \\ $M_{r}=303.71$ \\ Monoclinic, $P 2_{1} / c$ \\ $a=9.2724(5) \AA$ \\ $b=11.7943$ (6) \\ $c=24.4404(11) \AA$ \\ $\beta=99.180(2)^{\circ}$ \\ $V=2638.6(2) \AA^{3}$ \\ $Z=8$}

\section{Data collection}

Bruker-Nonius APEX2 CCD areadetector diffractometer $\varphi$ and $\omega$ scans

Absorption correction: multi-scan (SADABS; Sheldrick, 2003)

$T_{\min }=0.942, T_{\max }=0.959$

90190 measured reflections

\section{Refinement}

Refinement on $F^{2}$

$R\left[F^{2}>2 \sigma\left(F^{2}\right)\right]=0.034$

$w R\left(F^{2}\right)=0.095$

$S=1.08$

6497 reflections

440 parameters

Only H-atom coordinates refined

\author{
$D_{x}=1.529 \mathrm{Mg} \mathrm{m}^{-3}$ \\ Mo $K \alpha$ radiation \\ Cell parameters from 7840 \\ reflections \\ $\theta=2.2-27.4^{\circ}$ \\ $\mu=0.30 \mathrm{~mm}^{-1}$ \\ $T=100$ (2) K \\ Block, colourless \\ $0.20 \times 0.16 \times 0.14 \mathrm{~mm}$
}

6497 independent reflections 5110 reflections with $I>2 \sigma(I)$ $R_{\text {int }}=0.050$

$\theta_{\text {max }}=28.2^{\circ}$

$h=-12 \rightarrow 12$

$k=-15 \rightarrow 15$

$l=-32 \rightarrow 32$ 


\section{organic papers}

\section{Table 1}

Hydrogen-bond geometry $\left(\AA \AA^{\circ}{ }^{\circ}\right.$.

\begin{tabular}{lllll}
\hline$D-\mathrm{H} \cdots A$ & $D-\mathrm{H}$ & $\mathrm{H} \cdots A$ & $D \cdots A$ & $D-\mathrm{H} \cdots A$ \\
\hline $\mathrm{N} 2 A-\mathrm{H} 2 A \cdots \mathrm{N} 7 B^{\mathrm{i}}$ & $0.890(17)$ & $2.197(17)$ & $3.0771(17)$ & $169.9(14)$ \\
$\mathrm{N} 2 A-\mathrm{H} 2 B \cdots \mathrm{N} 1 B$ & $0.861(17)$ & $2.212(18)$ & $3.0634(16)$ & $169.9(15)$ \\
$\mathrm{N} 2 B-\mathrm{H} 2 D \cdots \mathrm{N} 3 A$ & $0.836(18)$ & $2.302(19)$ & $3.1378(17)$ & $177.8(17)$ \\
$\mathrm{N} 2 B-\mathrm{H} 2 D \cdots \mathrm{O} 10 A$ & $0.836(18)$ & $2.464(17)$ & $2.7501(15)$ & $101.1(13)$ \\
$\mathrm{N} 2 B-\mathrm{H} 2 C \cdots \mathrm{O} 10 A$ & $0.886(18)$ & $2.432(17)$ & $2.7501(15)$ & $101.6(13)$ \\
$\mathrm{C} 8 B-\mathrm{H} 8 B \cdots \mathrm{N} 7 A^{\mathrm{ii}}$ & $0.915(17)$ & $2.375(17)$ & $3.2779(18)$ & $169.1(14)$
\end{tabular}

Symmetry codes: (i) $2-x, y-\frac{1}{2}, \frac{1}{2}-z$; (ii) $1+x, 1+y, z$.

The coordinates of all $\mathrm{H}$ atoms were refined freely, whilst the isotropic displacement parameters were treated as riding on the bound atom such that $U_{\text {iso }}(\mathrm{H})=1.2 U_{\text {eq }}(\mathrm{C}, \mathrm{N})$.

Data collection: APEX2 (Bruker, 2003); cell refinement: SAINT (Bruker, 1998); data reduction: $S A I N T$; $\operatorname{program}(\mathrm{s})$ used to solve structure: SHELXS97 (Sheldrick, 1997); program(s) used to refine structure: SHELXL97 (Sheldrick, 1997); molecular graphics: SHELXTL (Bruker, 1998); software used to prepare material for publication: SHELXTL.
The authors thank Christina Graham for micro-analysis and the European Commission Framework 6 programme (project ref. LSHB-CT-2003-503480) for funding.

\section{References}

Bernstein, J., Davis, R. E., Shimoni, L. \& Chang, N.-L. (1995). Angew. Chem. Int. Ed. Engl. 34, 1555-1573.

Bruker (1998). SHELXTL (Version 5.1) and SAINT. Bruker AXS Inc., Madison, Wisconsin, USA.

Bruker (2003). APEX2. Version 1.0-8. Bruker AXS Inc., Madison, Wisconsin, USA.

Dey, S. \& Garner, P. (2000). J. Org. Chem. 65, 7697-7699.

Howarth, N. M., Lindsell, W. E., Murray, E. \& Preston, P. N. (2003). Tetrahedron Lett. 44, 8089-8092.

Howarth, N. M. \& Wakelin, L. P. G. (1997). J. Org. Chem. 62, 5441-5450.

Howarth, N. M., Wakelin, L. P. G. \& Walker, D. M. (2003). Tetrahedron Lett. 44, 695-698.

Sheldrick, G. M. (1997). SHELXS97 and SHELXL97. University of Göttingen, Germany.

Sheldrick, G. M. (2003). SADABS. University of Göttingen, Germany.

Vyle, J. S. \& Howarth, N. M. (2001). Specialist Periodical Reports, Organophosphorous Chemistry, Vol. 31, edited by D. W. Allen \& J. C. Tebby, pp. 135-218. London: Royal Society of Chemistry. 


\title{
supporting information
}

Acta Cryst. (2005). E61, o486-0488 [https://doi.org/10.1107/S1600536805002047]

\section{Benzyl 2-amino-6-chloro-9H-purine-9-carboxylate}

\author{
Kassim F. Adebambo, Nicola M. Howarth and Georgina M. Rosair
}

Benzyl 2-amino-6-chloro-9H-purine-9-carboxylate

Crystal data

$\mathrm{C}_{13} \mathrm{H}_{10} \mathrm{ClN}_{5} \mathrm{O}_{2}$

$M_{r}=303.71$

Monoclinic, $P 2_{1} / c$

$a=9.2724(5) \AA$

$b=11.7943(6) \AA$

$c=24.4404(11) \AA$

$\beta=99.180(2)^{\circ}$

$V=2638.6(2) \AA^{3}$

$Z=8$

$F(000)=1248$

$D_{\mathrm{x}}=1.529 \mathrm{Mg} \mathrm{m}^{-3}$

Mo $K \alpha$ radiation, $\lambda=0.71073 \AA$

Cell parameters from 7840 reflections

$\theta=2.2-27.4^{\circ}$

$\mu=0.30 \mathrm{~mm}^{-1}$

$T=100 \mathrm{~K}$

Block, colourless

$0.20 \times 0.16 \times 0.14 \mathrm{~mm}$

\section{Data collection}

Bruker-Nonius Apex2 CCD area-detector diffractometer

Radiation source: fine-focus sealed tube

Graphite monochromator

$\varphi$ and $\omega$ scans

Absorption correction: multi-scan

(SADABS; Sheldrick, 2003)

$T_{\min }=0.942, T_{\max }=0.959$

90190 measured reflections

6497 independent reflections

5110 reflections with $I>2 \sigma(I)$

$R_{\text {int }}=0.050$

$\theta_{\max }=28.2^{\circ}, \theta_{\min }=2.4^{\circ}$

$h=-12 \rightarrow 12$

$k=-15 \rightarrow 15$

$l=-32 \rightarrow 32$

\section{Refinement}

Refinement on $F^{2}$

Least-squares matrix: full

$R\left[F^{2}>2 \sigma\left(F^{2}\right)\right]=0.034$

$w R\left(F^{2}\right)=0.095$

$S=1.08$

6497 reflections

440 parameters

0 restraints

Primary atom site location: structure-invariant

direct methods

Secondary atom site location: difference Fourier map

Hydrogen site location: inferred from

neighbouring sites

Only $\mathrm{H}$-atom coordinates refined

$w=1 /\left[\sigma^{2}\left(F_{\mathrm{o}}^{2}\right)+(0.0493 P)^{2}+0.5642 P\right]$

where $P=\left(F_{\mathrm{o}}{ }^{2}+2 F_{\mathrm{c}}{ }^{2}\right) / 3$

$(\Delta / \sigma)_{\max }=0.001$

$\Delta \rho_{\max }=0.31 \mathrm{e} \AA^{-3}$

$\Delta \rho_{\min }=-0.26$ e $\AA^{-3}$

Special details

Geometry. All e.s.d.'s (except the e.s.d. in the dihedral angle between two 1.s. planes) are estimated using the full covariance matrix. The cell e.s.d.'s are taken into account individually in the estimation of e.s.d.'s in distances, angles and torsion angles; correlations between e.s.d.'s in cell parameters are only used when they are defined by crystal symmetry. An approximate (isotropic) treatment of cell e.s.d.'s is used for estimating e.s.d.'s involving 1.s. planes. 
Refinement. Refinement of $F^{2}$ against ALL reflections. The weighted $R$-factor $w R$ and goodness of fit $S$ are based on $F^{2}$, conventional $R$-factors $R$ are based on $F$, with $F$ set to zero for negative $F^{2}$. The threshold expression of $F^{2}>\sigma\left(F^{2}\right)$ is used only for calculating $R$-factors(gt) etc. and is not relevant to the choice of reflections for refinement. $R$-factors based on $F^{2}$ are statistically about twice as large as those based on $F$, and $R$ - factors based on ALL data will be even larger.

Fractional atomic coordinates and isotropic or equivalent isotropic displacement parameters $\left(\hat{A}^{2}\right)$

\begin{tabular}{|c|c|c|c|c|}
\hline & $x$ & $y$ & $z$ & $U_{\text {iso }} * / U_{\text {eq }}$ \\
\hline N1A & $0.61778(12)$ & $0.60392(9)$ & $0.27356(5)$ & $0.0221(2)$ \\
\hline $\mathrm{C} 2 \mathrm{~A}$ & $0.68744(14)$ & $0.68392(11)$ & $0.30916(5)$ & 0.0198 \\
\hline N2A & $0.79239(13)$ & $0.74276(10)$ & $0.29036(5)$ & $0.0216(2)$ \\
\hline $\mathrm{H} 2 \mathrm{~A}$ & $0.8060(17)$ & $0.7303(13)$ & $0.2556(7)$ & $0.026^{*}$ \\
\hline $\mathrm{H} 2 \mathrm{~B}$ & $0.8279(17)$ & $0.8019(15)$ & $0.3083(7)$ & $0.026^{*}$ \\
\hline N3A & $0.66031(12)$ & $0.70736(9)$ & $0.36113(5)$ & $0.0200(2)$ \\
\hline C4A & $0.55724(14)$ & $0.64099(11)$ & $0.37592(5)$ & $0.0205(3)$ \\
\hline C5A & $0.47945(14)$ & $0.55588(11)$ & $0.34388(6)$ & $0.0220(3)$ \\
\hline C6A & $0.51639(15)$ & $0.54252(11)$ & $0.29143(6)$ & $0.0235(3)$ \\
\hline $\mathrm{Cl6A}$ & $0.42563(4)$ & $0.44372(3)$ & $0.246400(16)$ & $0.03247(10)$ \\
\hline N7A & $0.38063(13)$ & $0.50166(10)$ & $0.37267(5)$ & $0.0257(3)$ \\
\hline C8A & $0.39612(15)$ & $0.55187(12)$ & $0.42032(6)$ & $0.0251(3)$ \\
\hline H8A & $0.3424(18)$ & $0.5360(14)$ & $0.4494(7)$ & $0.030^{*}$ \\
\hline N9A & $0.50257(12)$ & $0.63862(10)$ & $0.42625(5)$ & $0.0226(2)$ \\
\hline C10A & $0.53597(15)$ & $0.71210(12)$ & $0.47149(6)$ & $0.0232(3)$ \\
\hline $\mathrm{O} 10 \mathrm{~A}$ & $0.62625(11)$ & $0.78565(9)$ & $0.47466(4)$ & $0.0296(2)$ \\
\hline O11A & $0.45297(10)$ & $0.68712(8)$ & $0.50966(4)$ & $0.0253(2)$ \\
\hline C12A & $0.46867(17)$ & $0.76553(13)$ & $0.55606(6)$ & 0.0279 \\
\hline $\mathrm{H} 12 \mathrm{~A}$ & 0.5735 (19) & $0.7687(14)$ & $0.5731(7)$ & $0.033 *$ \\
\hline H12B & $0.4332(18)$ & $0.8442(15)$ & $0.5419(7)$ & $0.033 *$ \\
\hline C13A & $0.37689(15)$ & $0.72644(12)$ & $0.59783(6)$ & 0.0238 \\
\hline C14A & $0.39219(17)$ & $0.78581(14)$ & $0.64765(6)$ & 0.0298 \\
\hline H14A & 0.4569 (19) & $0.8464(15)$ & $0.6517(7)$ & $0.036^{*}$ \\
\hline C15A & $0.31117(18)$ & $0.75506(14)$ & $0.68822(7)$ & $0.0342(4)$ \\
\hline $\mathrm{H} 15 \mathrm{~A}$ & 0.3227 (19) & $0.7941(16)$ & $0.7231(8)$ & $0.041 *$ \\
\hline C16A & $0.21629(17)$ & $0.66388(14)$ & $0.68009(6)$ & $0.0313(3)$ \\
\hline H16A & 0.1622 (19) & $0.6419(14)$ & $0.7096(7)$ & $0.038 *$ \\
\hline C17A & $0.20041(16)$ & $0.60416(13)$ & $0.63074(6)$ & 0.0278 \\
\hline H17A & 0.1355 (19) & $0.5397(15)$ & $0.6259(7)$ & $0.033^{*}$ \\
\hline C18A & $0.28058(15)$ & $0.63531(12)$ & $0.58904(6)$ & $0.0247(3)$ \\
\hline H18A & $0.2728(17)$ & $0.5952(14)$ & $0.5553(7)$ & $0.030^{*}$ \\
\hline N1B & $0.89220(12)$ & $0.97073(9)$ & $0.34342(4)$ & $0.0214(2)$ \\
\hline $\mathrm{C} 2 \mathrm{~B}$ & $0.87924(15)$ & $0.99619(11)$ & $0.39669(5)$ & $0.0214(3)$ \\
\hline $\mathrm{N} 2 \mathrm{~B}$ & $0.79408(15)$ & $0.92729(11)$ & $0.42127(5)$ & 0.0281 \\
\hline $\mathrm{H} 2 \mathrm{C}$ & 0.7907 (19) & $0.9371(14)$ & $0.4570(8)$ & $0.034 *$ \\
\hline $\mathrm{H} 2 \mathrm{D}$ & 0.7575 (19) & $0.8698(16)$ & $0.4045(7)$ & $0.034 *$ \\
\hline N3B & $0.94051(12)$ & $1.08495(9)$ & $0.42677(5)$ & 0.0218 \\
\hline $\mathrm{C} 4 \mathrm{~B}$ & $1.01863(14)$ & $1.15002(11)$ & $0.39844(5)$ & 0.0199 \\
\hline C5B & $1.04168(14)$ & $1.13338(11)$ & $0.34371(5)$ & 0.0208 \\
\hline C6B & $0.97127(15)$ & $1.03962(11)$ & $0.31767(5)$ & 0.0207 \\
\hline
\end{tabular}




$\begin{array}{lllll}\text { C16B } & 0.97980(4) & 1.01065(3) & 0.248990(14) & 0.02927(10) \\ \text { N7B } & 1.12714(13) & 1.21991(10) & 0.32651(5) & 0.0235(2) \\ \text { C8B } & 1.15482(15) & 1.28696(12) & 0.36905(6) & 0.0231(3) \\ \text { H8B } & 1.2067(17) & 1.3530(14) & 0.3698(6) & 0.028^{*} \\ \text { N9B } & 1.09377(12) & 1.25019(9) & 0.41508(5) & 0.0212(2) \\ \text { C10B } & 1.09411(14) & 1.31502(11) & 0.46355(5) & 0.0213(3) \\ \text { O10B } & 1.15479(1) & 1.40544(8) & 0.47069(4) & 0.0289(2) \\ \text { O11B } & 1.01832(10) & 1.26210(8) & 0.49711(4) & 0.0226(2) \\ \text { C12B } & 1.00086(16) & 1.31964(13) & 0.54817(6) & 0.0251(3) \\ \text { H12C } & 1.0960(18) & 1.3284(14) & 0.5704(7) & 0.030^{*} \\ \text { H12D } & 0.9605(17) & 1.3948(15) & 0.5387(6) & 0.028(4)^{*} \\ \text { C13B } & 0.89917(15) & 1.24971(12) & 0.57672(6) & 0.0245(3) \\ \text { C14B } & 0.87699(18) & 1.28219(14) & 0.62963(6) & 0.0322(3) \\ \text { H14B } & 0.9291(19) & 1.3492(16) & 0.6463(7) & 0.039^{*} \\ \text { C15B } & 0.7849(2) & 1.22082(16) & 0.65775(7) & 0.0394(4) \\ \text { H15B } & 0.773(2) & 1.2449(16) & 0.6929(8) & 0.047^{*} \\ \text { C16B } & 0.71261(19) & 1.12573(17) & 0.63370(7) & 0.0411(4) \\ \text { H16B } & 0.651(2) & 1.0837(17) & 0.6524(8) & 0.049^{*} \\ \text { C17B } & 0.73360(18) & 1.09264(15) & 0.58100(7) & 0.0354(4) \\ \text { H17B } & 0.682(2) & 1.0251(16) & 0.5640(8) & 0.043^{*} \\ \text { C18B } & 0.82698(16) & 1.15414(13) & 0.55256(6) & 0.0278(3) \\ \text { H18B } & 0.8412(18) & 1.1321(14) & 0.5157(7) & 0.033^{*}\end{array}$

Atomic displacement parameters $\left(\AA^{2}\right)$

\begin{tabular}{lllllll}
\hline & $U^{11}$ & $U^{22}$ & $U^{33}$ & $U^{12}$ & $U^{13}$ & $U^{23}$ \\
\hline $\mathrm{N} 1 \mathrm{~A}$ & $0.0207(6)$ & $0.0198(5)$ & $0.0251(6)$ & $0.0002(4)$ & $0.0013(4)$ & $-0.0006(4)$ \\
$\mathrm{C} 2 \mathrm{~A}$ & $0.0191(6)$ & $0.0184(6)$ & $0.0215(6)$ & $0.0023(5)$ & $0.0023(5)$ & $0.0011(5)$ \\
$\mathrm{N} 2 \mathrm{~A}$ & $0.0245(6)$ & $0.0206(6)$ & $0.0208(6)$ & $-0.0034(5)$ & $0.0067(5)$ & $-0.0023(5)$ \\
$\mathrm{N} 3 \mathrm{~A}$ & $0.0195(5)$ & $0.0194(5)$ & $0.0213(5)$ & $-0.0020(4)$ & $0.0037(4)$ & $0.0012(4)$ \\
$\mathrm{C} 4 \mathrm{~A}$ & $0.0192(6)$ & $0.0194(6)$ & $0.0229(6)$ & $0.0008(5)$ & $0.0037(5)$ & $0.0027(5)$ \\
$\mathrm{C} 5 \mathrm{~A}$ & $0.0177(6)$ & $0.0190(6)$ & $0.0293(7)$ & $-0.0014(5)$ & $0.0037(5)$ & $0.0017(5)$ \\
$\mathrm{C} 6 \mathrm{~A}$ & $0.0203(7)$ & $0.0196(6)$ & $0.0289(7)$ & $0.0006(5)$ & $-0.0010(5)$ & $-0.0017(5)$ \\
$\mathrm{C} 6 \mathrm{~A}$ & $0.03002(19)$ & $0.02945(19)$ & $0.0366(2)$ & $-0.00807(15)$ & $0.00110(15)$ & $-0.00987(15)$ \\
$\mathrm{N} 7 \mathrm{~A}$ & $0.0202(6)$ & $0.0228(6)$ & $0.0347(7)$ & $-0.0033(5)$ & $0.0065(5)$ & $0.0016(5)$ \\
$\mathrm{C} 8 \mathrm{~A}$ & $0.0212(7)$ & $0.0208(7)$ & $0.0340(8)$ & $-0.0038(5)$ & $0.0069(6)$ & $0.0033(6)$ \\
N9A & $0.0210(6)$ & $0.0230(6)$ & $0.0244(6)$ & $-0.0050(5)$ & $0.0059(4)$ & $0.0016(4)$ \\
$\mathrm{C} 10 \mathrm{~A}$ & $0.0198(7)$ & $0.0272(7)$ & $0.0232(7)$ & $-0.0014(5)$ & $0.0049(5)$ & $0.0049(5)$ \\
O10A & $0.0298(5)$ & $0.0343(6)$ & $0.0256(5)$ & $-0.0129(5)$ & $0.0074(4)$ & $-0.0011(4)$ \\
O11A & $0.0252(5)$ & $0.0288(5)$ & $0.0233(5)$ & $-0.0062(4)$ & $0.0080(4)$ & $0.0009(4)$ \\
C12A & $0.0286(8)$ & $0.0328(8)$ & $0.0230(7)$ & $-0.0079(6)$ & $0.0058(6)$ & $-0.0008(6)$ \\
C13A & $0.0204(7)$ & $0.0273(7)$ & $0.0240(7)$ & $0.0005(5)$ & $0.0042(5)$ & $0.0045(5)$ \\
C14A & $0.0289(8)$ & $0.0333(8)$ & $0.0276(7)$ & $-0.0075(6)$ & $0.0057(6)$ & $0.0002(6)$ \\
C15A & $0.0362(9)$ & $0.0422(9)$ & $0.0259(8)$ & $-0.0039(7)$ & $0.0108(7)$ & $-0.0025(7)$ \\
C16A & $0.0288(8)$ & $0.0370(8)$ & $0.0302(8)$ & $-0.0001(6)$ & $0.0115(6)$ & $0.0060(6)$ \\
C17A & $0.0221(7)$ & $0.0278(7)$ & $0.0345(8)$ & $-0.0009(6)$ & $0.0077(6)$ & $0.0053(6)$ \\
C18A & $0.0210(7)$ & $0.0273(7)$ & $0.0260(7)$ & $0.0011(5)$ & $0.0045(5)$ & $0.0026(6)$ \\
N1B & $0.0248(6)$ & $0.0194(5)$ & $0.0206(5)$ & $-0.0009(4)$ & $0.0055(4)$ & $-0.0006(4)$ \\
& & & & & &
\end{tabular}




\begin{tabular}{lllllll} 
& & & & & \\
$\mathrm{C} 2 \mathrm{~B}$ & $0.0244(7)$ & $0.0184(6)$ & $0.0222(6)$ & $-0.0012(5)$ & $0.0055(5)$ & $0.0006(5)$ \\
$\mathrm{N} 2 \mathrm{~B}$ & $0.0392(7)$ & $0.0243(6)$ & $0.0232(6)$ & $-0.0124(5)$ & $0.0124(5)$ & $-0.0036(5)$ \\
$\mathrm{N} 3 \mathrm{~B}$ & $0.0264(6)$ & $0.0185(5)$ & $0.0216(5)$ & $-0.0040(4)$ & $0.0069(5)$ & $0.0004(4)$ \\
$\mathrm{C} 4 \mathrm{~B}$ & $0.0205(6)$ & $0.0179(6)$ & $0.0213(6)$ & $-0.0009(5)$ & $0.0035(5)$ & $-0.0001(5)$ \\
$\mathrm{C} 5 \mathrm{~B}$ & $0.0226(7)$ & $0.0190(6)$ & $0.0219(6)$ & $-0.0001(5)$ & $0.0068(5)$ & $0.0026(5)$ \\
$\mathrm{C} 6 \mathrm{~B}$ & $0.0237(7)$ & $0.0197(6)$ & $0.0194(6)$ & $0.0011(5)$ & $0.0057(5)$ & $0.0001(5)$ \\
C16B & $0.0416(2)$ & $0.02730(18)$ & $0.02115(16)$ & $-0.00514(15)$ & $0.01167(14)$ & $-0.00339(13)$ \\
N7B & $0.0244(6)$ & $0.0220(6)$ & $0.0253(6)$ & $-0.0037(5)$ & $0.0075(5)$ & $0.0017(5)$ \\
C8B & $0.0234(7)$ & $0.0212(7)$ & $0.0258(7)$ & $-0.0036(5)$ & $0.0074(5)$ & $0.0029(5)$ \\
N9B & $0.0232(6)$ & $0.0192(5)$ & $0.0218(6)$ & $-0.0044(4)$ & $0.0055(5)$ & $-0.0003(4)$ \\
C10B & $0.0195(6)$ & $0.0206(6)$ & $0.0232(6)$ & $-0.0007(5)$ & $0.0021(5)$ & $0.0013(5)$ \\
O10B & $0.0322(6)$ & $0.0224(5)$ & $0.0330(6)$ & $-0.0078(4)$ & $0.0077(4)$ & $-0.0042(4)$ \\
O11B & $0.0265(5)$ & $0.0227(5)$ & $0.0192(5)$ & $-0.0049(4)$ & $0.0053(4)$ & $-0.0028(4)$ \\
C12B & $0.0278(7)$ & $0.0258(7)$ & $0.0212(7)$ & $0.0000(6)$ & $0.0027(6)$ & $-0.0065(5)$ \\
C13B & $0.0230(7)$ & $0.0285(7)$ & $0.0214(7)$ & $0.0069(6)$ & $0.0018(5)$ & $0.0012(5)$ \\
C14B & $0.0357(9)$ & $0.0368(9)$ & $0.0238(7)$ & $0.0123(7)$ & $0.0034(6)$ & $-0.0014(6)$ \\
C15B & $0.0439(10)$ & $0.0508(10)$ & $0.0262(8)$ & $0.0200(8)$ & $0.0140(7)$ & $0.0065(7)$ \\
C16B & $0.0370(9)$ & $0.0504(11)$ & $0.0405(9)$ & $0.0116(8)$ & $0.0201(7)$ & $0.0167(8)$ \\
C17B & $0.0304(8)$ & $0.0390(9)$ & $0.0389(9)$ & $-0.0006(7)$ & $0.0116(7)$ & $0.0066(7)$ \\
C18B & $0.0256(7)$ & $0.0324(8)$ & $0.0262(7)$ & $0.0007(6)$ & $0.0062(6)$ & $0.0007(6)$ \\
& & & & & & \\
\hline
\end{tabular}

Geometric parameters $\left(\AA,{ }^{o}\right)$

\begin{tabular}{llll}
\hline N1A-C6A & $1.3153(18)$ & $\mathrm{N} 1 \mathrm{~B}-\mathrm{C} 6 \mathrm{~B}$ & $1.3195(17)$ \\
$\mathrm{N} 1 \mathrm{~A}-\mathrm{C} 2 \mathrm{~A}$ & $1.3730(17)$ & $\mathrm{N} 1 \mathrm{~B}-\mathrm{C} 2 \mathrm{~B}$ & $1.3600(17)$ \\
$\mathrm{C} 2 \mathrm{~A}-\mathrm{N} 2 \mathrm{~A}$ & $1.3350(17)$ & $\mathrm{C} 2 \mathrm{~B}-\mathrm{N} 2 \mathrm{~B}$ & $1.3403(17)$ \\
$\mathrm{C} 2 \mathrm{~A}-\mathrm{N} 3 \mathrm{~A}$ & $1.3620(17)$ & $\mathrm{C} 2 \mathrm{~B}-\mathrm{N} 3 \mathrm{~B}$ & $1.3513(17)$ \\
$\mathrm{N} 2 \mathrm{~A}-\mathrm{H} 2 \mathrm{~A}$ & $0.890(17)$ & $\mathrm{N} 2 \mathrm{~B}-\mathrm{H} 2 \mathrm{C}$ & $0.886(18)$ \\
$\mathrm{N} 2 \mathrm{~A}-\mathrm{H} 2 \mathrm{~B}$ & $0.861(17)$ & $\mathrm{N} 2 \mathrm{~B}-\mathrm{H} 2 \mathrm{D}$ & $0.836(18)$ \\
$\mathrm{N} 3 \mathrm{~A}-\mathrm{C} 4 \mathrm{~A}$ & $1.3292(17)$ & $\mathrm{N} 3 \mathrm{~B}-\mathrm{C} 4 \mathrm{~B}$ & $1.3240(17)$ \\
$\mathrm{C} 4 \mathrm{~A}-\mathrm{C} 5 \mathrm{~A}$ & $1.4003(18)$ & $\mathrm{C} 4 \mathrm{~B}-\mathrm{N} 9 \mathrm{~B}$ & $1.3986(17)$ \\
$\mathrm{C} 4 \mathrm{~A}-\mathrm{N} 9 \mathrm{~A}$ & $1.4036(17)$ & $\mathrm{C} 4 \mathrm{~B}-\mathrm{C} 5 \mathrm{~B}$ & $1.4017(18)$ \\
$\mathrm{C} 5 \mathrm{~A}-\mathrm{C} 6 \mathrm{~A}$ & $1.388(2)$ & $\mathrm{C} 5 \mathrm{~B}-\mathrm{C} 6 \mathrm{~B}$ & $1.3863(19)$ \\
$\mathrm{C} 5 \mathrm{~A}-\mathrm{N} 7 \mathrm{~A}$ & $1.3962(17)$ & $\mathrm{C} 5 \mathrm{~B}-\mathrm{N} 7 \mathrm{~B}$ & $1.3973(17)$ \\
$\mathrm{C} 6 \mathrm{~A}-\mathrm{C} 16 \mathrm{~A}$ & $1.7267(14)$ & $\mathrm{C} 6 \mathrm{~B}-\mathrm{C} 16 \mathrm{~B}$ & $1.7271(13)$ \\
$\mathrm{N} 7 \mathrm{~A}-\mathrm{C} 8 \mathrm{~A}$ & $1.2940(19)$ & $\mathrm{N} 7 \mathrm{~B}-\mathrm{C} 8 \mathrm{~B}$ & $1.2988(18)$ \\
$\mathrm{C} 8 \mathrm{~A}-\mathrm{N} 9 \mathrm{~A}$ & $1.4130(17)$ & $\mathrm{C} 8 \mathrm{~B}-\mathrm{N} 9 \mathrm{~B}$ & $1.4062(17)$ \\
$\mathrm{C} 8 \mathrm{~A}-\mathrm{H} 8 \mathrm{~A}$ & $0.950(17)$ & $\mathrm{C} 8 \mathrm{~B}-\mathrm{H} 8 \mathrm{~B}$ & $0.915(17)$ \\
N9A-C10A & $1.4000(18)$ & $\mathrm{N} 9 \mathrm{~B}-\mathrm{C} 10 \mathrm{~B}$ & $1.4095(17)$ \\
$\mathrm{C} 10 \mathrm{~A}-\mathrm{O} 10 \mathrm{~A}$ & $1.1994(17)$ & $\mathrm{C} 10 \mathrm{~B}-\mathrm{O} 10 \mathrm{~B}$ & $1.2054(16)$ \\
$\mathrm{C} 10 \mathrm{~A}-\mathrm{O} 11 \mathrm{~A}$ & $1.3336(16)$ & $\mathrm{C} 10 \mathrm{~B}-\mathrm{O} 11 \mathrm{~B}$ & $1.3192(16)$ \\
$\mathrm{O} 11 \mathrm{~A}-\mathrm{C} 12 \mathrm{~A}$ & $1.4525(18)$ & $\mathrm{O} 11 \mathrm{~B}-\mathrm{C} 12 \mathrm{~B}$ & $1.4523(16)$ \\
$\mathrm{C} 12 \mathrm{~A}-\mathrm{C} 13 \mathrm{~A}$ & $1.502(2)$ & $\mathrm{C} 12 \mathrm{~B}-\mathrm{C} 13 \mathrm{~B}$ & $1.505(2)$ \\
$\mathrm{C} 12 \mathrm{~A}-\mathrm{H} 12 \mathrm{~A}$ & $0.995(18)$ & $\mathrm{C} 12 \mathrm{~B}-\mathrm{H} 12 \mathrm{C}$ & $0.965(17)$ \\
$\mathrm{C} 12 \mathrm{~A}-\mathrm{H} 12 \mathrm{~B}$ & $1.026(18)$ & $\mathrm{C} 12 \mathrm{~B}-\mathrm{H} 12 \mathrm{D}$ & $0.975(17)$ \\
$\mathrm{C} 13 \mathrm{~A}-\mathrm{C} 18 \mathrm{~A}$ & $1.392(2)$ & $\mathrm{C} 13 \mathrm{~B}-\mathrm{C} 18 \mathrm{~B}$ & $1.393(2)$ \\
$\mathrm{C} 13 \mathrm{~A}-\mathrm{C} 14 \mathrm{~A}$ & $1.392(2)$ & $\mathrm{C} 13 \mathrm{~B}-\mathrm{C} 14 \mathrm{~B}$ & $1.395(2)$ \\
$\mathrm{C} 14 \mathrm{~A}-\mathrm{C} 15 \mathrm{~A}$ & $1.384(2)$ & $\mathrm{C} 14 \mathrm{~B}-\mathrm{C} 15 \mathrm{~B}$ & $1.383(2)$
\end{tabular}


C14A-H14A
C15A-C16A
C15A-H15A
C16A-C17A
C16A-H16A
C17A-C18A
C17A-H17A
C18A-H18A

$\mathrm{C} 6 \mathrm{~A}-\mathrm{N} 1 \mathrm{~A}-\mathrm{C} 2 \mathrm{~A}$

$\mathrm{N} 2 \mathrm{~A}-\mathrm{C} 2 \mathrm{~A}-\mathrm{N} 3 \mathrm{~A}$

$\mathrm{N} 2 \mathrm{~A}-\mathrm{C} 2 \mathrm{~A}-\mathrm{N} 1 \mathrm{~A}$

$\mathrm{N} 3 \mathrm{~A}-\mathrm{C} 2 \mathrm{~A}-\mathrm{N} 1 \mathrm{~A}$

$\mathrm{C} 2 \mathrm{~A}-\mathrm{N} 2 \mathrm{~A}-\mathrm{H} 2 \mathrm{~A}$

$\mathrm{C} 2 \mathrm{~A}-\mathrm{N} 2 \mathrm{~A}-\mathrm{H} 2 \mathrm{~B}$

$\mathrm{H} 2 \mathrm{~A}-\mathrm{N} 2 \mathrm{~A}-\mathrm{H} 2 \mathrm{~B}$

$\mathrm{C} 4 \mathrm{~A}-\mathrm{N} 3 \mathrm{~A}-\mathrm{C} 2 \mathrm{~A}$

$\mathrm{N} 3 \mathrm{~A}-\mathrm{C} 4 \mathrm{~A}-\mathrm{C} 5 \mathrm{~A}$

$\mathrm{N} 3 \mathrm{~A}-\mathrm{C} 4 \mathrm{~A}-\mathrm{N} 9 \mathrm{~A}$

$\mathrm{C} 5 \mathrm{~A}-\mathrm{C} 4 \mathrm{~A}-\mathrm{N} 9 \mathrm{~A}$

$\mathrm{C} 6 \mathrm{~A}-\mathrm{C} 5 \mathrm{~A}-\mathrm{N} 7 \mathrm{~A}$

$\mathrm{C} 6 \mathrm{~A}-\mathrm{C} 5 \mathrm{~A}-\mathrm{C} 4 \mathrm{~A}$

$\mathrm{N} 7 \mathrm{~A}-\mathrm{C} 5 \mathrm{~A}-\mathrm{C} 4 \mathrm{~A}$

$\mathrm{N} 1 \mathrm{~A}-\mathrm{C} 6 \mathrm{~A}-\mathrm{C} 5 \mathrm{~A}$

N1A-C6A-Cl6A

$\mathrm{C} 5 \mathrm{~A}-\mathrm{C} 6 \mathrm{~A}-\mathrm{Cl} 6 \mathrm{~A}$

$\mathrm{C} 8 \mathrm{~A}-\mathrm{N} 7 \mathrm{~A}-\mathrm{C} 5 \mathrm{~A}$

$\mathrm{N} 7 \mathrm{~A}-\mathrm{C} 8 \mathrm{~A}-\mathrm{N} 9 \mathrm{~A}$

$\mathrm{N} 7 \mathrm{~A}-\mathrm{C} 8 \mathrm{~A}-\mathrm{H} 8 \mathrm{~A}$

$\mathrm{N} 9 \mathrm{~A}-\mathrm{C} 8 \mathrm{~A}-\mathrm{H} 8 \mathrm{~A}$

C10A-N9A-C4A

C10A-N9A-C8A

C4A-N9A-C8A

O10A-C10A-O11A

O10A-C10A-N9A

O11A-C10A-N9A

$\mathrm{C} 10 \mathrm{~A}-\mathrm{O} 11 \mathrm{~A}-\mathrm{C} 12 \mathrm{~A}$

O11A-C12A-C13A

$\mathrm{O} 11 \mathrm{~A}-\mathrm{C} 12 \mathrm{~A}-\mathrm{H} 12 \mathrm{~A}$

$\mathrm{C} 13 \mathrm{~A}-\mathrm{C} 12 \mathrm{~A}-\mathrm{H} 12 \mathrm{~A}$

O11A-C12A-H12B

$\mathrm{C} 13 \mathrm{~A}-\mathrm{C} 12 \mathrm{~A}-\mathrm{H} 12 \mathrm{~B}$

H12A-C12A-H12B

$\mathrm{C} 18 \mathrm{~A}-\mathrm{C} 13 \mathrm{~A}-\mathrm{C} 14 \mathrm{~A}$

C18A-C13A-C12A

$\mathrm{C} 14 \mathrm{~A}-\mathrm{C} 13 \mathrm{~A}-\mathrm{C} 12 \mathrm{~A}$

$\mathrm{C} 15 \mathrm{~A}-\mathrm{C} 14 \mathrm{~A}-\mathrm{C} 13 \mathrm{~A}$

C15A-C14A-H14A
$0.928(18)$

$1.384(2)$

0.959 (19)

$1.384(2)$

$0.976(18)$

$1.403(2)$

$0.965(18)$

$0.943(17)$

$117.19(12)$

$117.95(12)$

$115.74(12)$

$126.31(12)$

$117.7(10)$

$119.1(11)$

$121.2(15)$

$112.45(11)$

$126.78(12)$

$128.75(12)$

104.47 (11)

$133.79(13)$

$114.64(12)$

$111.57(12)$

$122.61(12)$

$117.48(11)$

$119.90(11)$

$104.86(12)$

$113.52(12)$

$126.0(10)$

$120.5(10)$

$127.92(11)$

$126.30(11)$

105.58 (11)

$126.10(13)$

$124.26(12)$

$109.64(11)$

$114.11(11)$

109.77 (12)

$108.6(10)$

$110.5(10)$

$109.0(9)$

$108.8(9)$

$110.2(13)$

$119.79(13)$

$123.44(13)$

$116.77(13)$

$120.23(15)$

122.7 (11)

$$
\begin{aligned}
& \text { C14B-H14B } \\
& \text { C15B-C16B } \\
& \text { C15B-H15B } \\
& \text { C16B-C17B } \\
& \text { C16B-H16B } \\
& \text { C17B-C18B } \\
& \text { C17B-H17B } \\
& \text { C18B-H18B }
\end{aligned}
$$

$\mathrm{C} 6 \mathrm{~B}-\mathrm{N} 1 \mathrm{~B}-\mathrm{C} 2 \mathrm{~B}$

$\mathrm{N} 2 \mathrm{~B}-\mathrm{C} 2 \mathrm{~B}-\mathrm{N} 3 \mathrm{~B}$

$\mathrm{N} 2 \mathrm{~B}-\mathrm{C} 2 \mathrm{~B}-\mathrm{N} 1 \mathrm{~B}$

$\mathrm{N} 3 \mathrm{~B}-\mathrm{C} 2 \mathrm{~B}-\mathrm{N} 1 \mathrm{~B}$

$\mathrm{C} 2 \mathrm{~B}-\mathrm{N} 2 \mathrm{~B}-\mathrm{H} 2 \mathrm{C}$

$\mathrm{C} 2 \mathrm{~B}-\mathrm{N} 2 \mathrm{~B}-\mathrm{H} 2 \mathrm{D}$

$\mathrm{H} 2 \mathrm{C}-\mathrm{N} 2 \mathrm{~B}-\mathrm{H} 2 \mathrm{D}$

$\mathrm{C} 4 \mathrm{~B}-\mathrm{N} 3 \mathrm{~B}-\mathrm{C} 2 \mathrm{~B}$

$\mathrm{N} 3 \mathrm{~B}-\mathrm{C} 4 \mathrm{~B}-\mathrm{N} 9 \mathrm{~B}$

$\mathrm{N} 3 \mathrm{~B}-\mathrm{C} 4 \mathrm{~B}-\mathrm{C} 5 \mathrm{~B}$

$\mathrm{N} 9 \mathrm{~B}-\mathrm{C} 4 \mathrm{~B}-\mathrm{C} 5 \mathrm{~B}$

$\mathrm{C} 6 \mathrm{~B}-\mathrm{C} 5 \mathrm{~B}-\mathrm{N} 7 \mathrm{~B}$

$\mathrm{C} 6 \mathrm{~B}-\mathrm{C} 5 \mathrm{~B}-\mathrm{C} 4 \mathrm{~B}$

$\mathrm{N} 7 \mathrm{~B}-\mathrm{C} 5 \mathrm{~B}-\mathrm{C} 4 \mathrm{~B}$

$\mathrm{N} 1 \mathrm{~B}-\mathrm{C} 6 \mathrm{~B}-\mathrm{C} 5 \mathrm{~B}$

$\mathrm{N} 1 \mathrm{~B}-\mathrm{C} 6 \mathrm{~B}-\mathrm{Cl} 6 \mathrm{~B}$

$\mathrm{C} 5 \mathrm{~B}-\mathrm{C} 6 \mathrm{~B}-\mathrm{Cl} 6 \mathrm{~B}$

$\mathrm{C} 8 \mathrm{~B}-\mathrm{N} 7 \mathrm{~B}-\mathrm{C} 5 \mathrm{~B}$

$\mathrm{N} 7 \mathrm{~B}-\mathrm{C} 8 \mathrm{~B}-\mathrm{N} 9 \mathrm{~B}$

$\mathrm{N} 7 \mathrm{~B}-\mathrm{C} 8 \mathrm{~B}-\mathrm{H} 8 \mathrm{~B}$

$\mathrm{N} 9 \mathrm{~B}-\mathrm{C} 8 \mathrm{~B}-\mathrm{H} 8 \mathrm{~B}$

$\mathrm{C} 4 \mathrm{~B}-\mathrm{N} 9 \mathrm{~B}-\mathrm{C} 8 \mathrm{~B}$

$\mathrm{C} 4 \mathrm{~B}-\mathrm{N} 9 \mathrm{~B}-\mathrm{C} 10 \mathrm{~B}$

$\mathrm{C} 8 \mathrm{~B}-\mathrm{N} 9 \mathrm{~B}-\mathrm{C} 10 \mathrm{~B}$

$\mathrm{O} 10 \mathrm{~B}-\mathrm{C} 10 \mathrm{~B}-\mathrm{O} 11 \mathrm{~B}$

O10B $-\mathrm{C} 10 \mathrm{~B}-\mathrm{N} 9 \mathrm{~B}$

O11B $-\mathrm{C} 10 \mathrm{~B}-\mathrm{N} 9 \mathrm{~B}$

$\mathrm{C} 10 \mathrm{~B}-\mathrm{O} 11 \mathrm{~B}-\mathrm{C} 12 \mathrm{~B}$

$\mathrm{O} 11 \mathrm{~B}-\mathrm{C} 12 \mathrm{~B}-\mathrm{C} 13 \mathrm{~B}$

$\mathrm{O} 11 \mathrm{~B}-\mathrm{C} 12 \mathrm{~B}-\mathrm{H} 12 \mathrm{C}$

$\mathrm{C} 13 \mathrm{~B}-\mathrm{C} 12 \mathrm{~B}-\mathrm{H} 12 \mathrm{C}$

$\mathrm{O} 11 \mathrm{~B}-\mathrm{C} 12 \mathrm{~B}-\mathrm{H} 12 \mathrm{D}$

$\mathrm{C} 13 \mathrm{~B}-\mathrm{C} 12 \mathrm{~B}-\mathrm{H} 12 \mathrm{D}$

$\mathrm{H} 12 \mathrm{C}-\mathrm{C} 12 \mathrm{~B}-\mathrm{H} 12 \mathrm{D}$

$\mathrm{C} 18 \mathrm{~B}-\mathrm{C} 13 \mathrm{~B}-\mathrm{C} 14 \mathrm{~B}$

$\mathrm{C} 18 \mathrm{~B}-\mathrm{C} 13 \mathrm{~B}-\mathrm{C} 12 \mathrm{~B}$

$\mathrm{C} 14 \mathrm{~B}-\mathrm{C} 13 \mathrm{~B}-\mathrm{C} 12 \mathrm{~B}$

$\mathrm{C} 15 \mathrm{~B}-\mathrm{C} 14 \mathrm{~B}-\mathrm{C} 13 \mathrm{~B}$

$\mathrm{C} 15 \mathrm{~B}-\mathrm{C} 14 \mathrm{~B}-\mathrm{H} 14 \mathrm{~B}$
$0.980(18)$

$1.388(3)$

$0.93(2)$

1.389 (2)

$0.93(2)$

1.397 (2)

0.984 (19)

0.968 (17)

$117.47(11)$

$116.90(12)$

$116.33(12)$

$126.75(12)$

$118.6(11)$

$119.6(12)$

120.9 (16)

$112.46(11)$

$128.77(12)$

$126.68(12)$

104.54 (11)

$133.84(12)$

$114.68(11)$

$111.41(11)$

$121.94(12)$

$117.18(10)$

$120.85(10)$

$104.66(11)$

$113.53(12)$

124.8 (10)

$121.6(10)$

105.87 (11)

$129.56(11)$

$123.90(11)$

$127.68(13)$

$122.69(12)$

$109.62(11)$

$117.18(11)$

107.83 (11)

108.5 (10)

112.4 (10)

108.2 (9)

111.3 (10)

$108.6(14)$

118.88 (14)

$122.76(13)$

$118.36(13)$

$120.72(16)$

$121.2(10)$ 


$\begin{array}{ll}\text { C13A-C14A-H14A } & 117.0(11) \\ \text { C16A-C15A-C14A } & 120.40(15) \\ \text { C16A-C15A-H15A } & 118.6(11) \\ \text { C14A-C15A-H15A } & 120.9(11) \\ \text { C15A-C16A-C17A } & 119.83(14) \\ \text { C15A-C16A-H16A } & 119.3(10) \\ \text { C17A-C16A-H16A } & 120.8(10) \\ \text { C16A-C17A-C18A } & 120.31(14) \\ \text { C16A-C17A-H17A } & 119.0(10) \\ \text { C18A-C17A-H17A } & 120.6(10) \\ \text { C13A-C18A-C17A } & 119.42(14) \\ \text { C13A-C18A-H18A } & 118.7(10) \\ \text { C17A-C18A-H18A } & 121.8(10) \\ & \\ \text { C6A-N1A-C2A-N2A } & 178.30(12) \\ \text { C6A-N1A-C2A-N3A } & -1.07(19) \\ \text { N2A-C2A-N3A-C4A } & -177.89(12) \\ \text { N1A-C2A-N3A-C4A } & 1.47(18) \\ \text { C2A-N3A-C4A-C5A } & -0.68(19) \\ \text { C2A-N3A-C4A-N9A } & 178.64(12) \\ \text { N3A-C4A-C5A-C6A } & -0.4(2) \\ \text { N9A-C4A-C5A-C6A } & -179.85(11) \\ \text { N3A-C4A-C5A-N7A } & 179.15(12) \\ \text { N9A-C4A-C5A-N7A } & -0.31(15) \\ \text { C2A-N1A-C6A-C5A } & -0.22(19) \\ \text { C2A-N1A-C6A-C16A } & 178.70(9) \\ \text { N7A-C5A-C6A-N1A } & -178.53(14) \\ \text { C4A-C5A-C6A-N1A } & 0.87(19) \\ \text { N7A-C5A-C6A-C16A } & 2.6(2) \\ \text { C4A-C5A-C6A-C16A } & -178.02(10) \\ \text { C6A-C5A-N7A-C8A } & 179.87(15) \\ \text { C4A-C5A-N7A-C8A } & 0.45(15) \\ \text { C5A-N7A-C8A-N9A } & -0.41(16) \\ \text { N3A-C4A-N9A-C10A } & 5.5(2) \\ \text { C5A-C4A-N9A-C10A } & -175.06(13) \\ \text { N3A-C4A-N9A-C8A } & -179.39(13) \\ \text { C5A-C4A-N9A-C8A } & 0.06(14) \\ \text { N7A-C8A-N9A-C10A } & 175.45(13) \\ \text { N7A-C8A-N9A-C4A } & 0.23(16) \\ \text { C4A-N9A-C10A-O10A } & -5.0(2) \\ \text { C8A-N9A-C10A-O10A } & -179.14(14) \\ \text { C4A-N9A-C10A-O11A } & 175.20(12) \\ \text { C8A-N9A-C10A-O11A } & 1.04(19) \\ \text { O10A-C10A-O11A-C12A } & 5.9(2) \\ \text { N9A-C10A-O11A-C12A } & -174.26(11) \\ \text { C10A-O11A-C12A-C13A } & -177.07(12) \\ \text { O11A-C12A-C13A-C18A } & -6.4(2) \\ \text { O11A-C12A-C13A-C14A } & 173.23(12)\end{array}$

$$
\begin{aligned}
& \text { C13B-C14B-H14B } \\
& \text { C14B-C15B-C16B } \\
& \text { C14B-C15B-H15B } \\
& \text { C16B-C15B-H15B } \\
& \text { C15B-C16B-C17B } \\
& \text { C15B-C16B-H16B } \\
& \text { C17B-C16B-H16B } \\
& \text { C16B-C17B-C18B } \\
& \text { C16B-C17B-H17B } \\
& \text { C18B-C17B-H17B } \\
& \text { C13B-C18B-C17B } \\
& \text { C13B-C18B-H18B } \\
& \text { C17B-C18B-H18B }
\end{aligned}
$$$$
\mathrm{C} 6 \mathrm{~B}-\mathrm{N} 1 \mathrm{~B}-\mathrm{C} 2 \mathrm{~B}-\mathrm{N} 2 \mathrm{~B}
$$$$
\mathrm{C} 6 \mathrm{~B}-\mathrm{N} 1 \mathrm{~B}-\mathrm{C} 2 \mathrm{~B}-\mathrm{N} 3 \mathrm{~B}
$$$$
\mathrm{N} 2 \mathrm{~B}-\mathrm{C} 2 \mathrm{~B}-\mathrm{N} 3 \mathrm{~B}-\mathrm{C} 4 \mathrm{~B}
$$$$
\mathrm{N} 1 \mathrm{~B}-\mathrm{C} 2 \mathrm{~B}-\mathrm{N} 3 \mathrm{~B}-\mathrm{C} 4 \mathrm{~B}
$$$$
\mathrm{C} 2 \mathrm{~B}-\mathrm{N} 3 \mathrm{~B}-\mathrm{C} 4 \mathrm{~B}-\mathrm{N} 9 \mathrm{~B}
$$$$
\mathrm{C} 2 \mathrm{~B}-\mathrm{N} 3 \mathrm{~B}-\mathrm{C} 4 \mathrm{~B}-\mathrm{C} 5 \mathrm{~B}
$$$$
\mathrm{N} 3 \mathrm{~B}-\mathrm{C} 4 \mathrm{~B}-\mathrm{C} 5 \mathrm{~B}-\mathrm{C} 6 \mathrm{~B}
$$$$
\mathrm{N} 9 \mathrm{~B}-\mathrm{C} 4 \mathrm{~B}-\mathrm{C} 5 \mathrm{~B}-\mathrm{C} 6 \mathrm{~B}
$$$$
\mathrm{N} 3 \mathrm{~B}-\mathrm{C} 4 \mathrm{~B}-\mathrm{C} 5 \mathrm{~B}-\mathrm{N} 7 \mathrm{~B}
$$$$
\mathrm{N} 9 \mathrm{~B}-\mathrm{C} 4 \mathrm{~B}-\mathrm{C} 5 \mathrm{~B}-\mathrm{N} 7 \mathrm{~B}
$$$$
\mathrm{C} 2 \mathrm{~B}-\mathrm{N} 1 \mathrm{~B}-\mathrm{C} 6 \mathrm{~B}-\mathrm{C} 5 \mathrm{~B}
$$$$
\mathrm{C} 2 \mathrm{~B}-\mathrm{N} 1 \mathrm{~B}-\mathrm{C} 6 \mathrm{~B}-\mathrm{Cl} 6 \mathrm{~B}
$$$$
\mathrm{N} 7 \mathrm{~B}-\mathrm{C} 5 \mathrm{~B}-\mathrm{C} 6 \mathrm{~B}-\mathrm{N} 1 \mathrm{~B}
$$$$
\mathrm{C} 4 \mathrm{~B}-\mathrm{C} 5 \mathrm{~B}-\mathrm{C} 6 \mathrm{~B}-\mathrm{N} 1 \mathrm{~B}
$$$$
\mathrm{N} 7 \mathrm{~B}-\mathrm{C} 5 \mathrm{~B}-\mathrm{C} 6 \mathrm{~B}-\mathrm{C} 6 \mathrm{~B}
$$$$
\mathrm{C} 4 \mathrm{~B}-\mathrm{C} 5 \mathrm{~B}-\mathrm{C} 6 \mathrm{~B}-\mathrm{Cl6B}
$$$$
\mathrm{C} 6 \mathrm{~B}-\mathrm{C} 5 \mathrm{~B}-\mathrm{N} 7 \mathrm{~B}-\mathrm{C} 8 \mathrm{~B}
$$$$
\mathrm{C} 4 \mathrm{~B}-\mathrm{C} 5 \mathrm{~B}-\mathrm{N} 7 \mathrm{~B}-\mathrm{C} 8 \mathrm{~B}
$$$$
\mathrm{C} 5 \mathrm{~B}-\mathrm{N} 7 \mathrm{~B}-\mathrm{C} 8 \mathrm{~B}-\mathrm{N} 9 \mathrm{~B}
$$$$
\mathrm{N} 3 \mathrm{~B}-\mathrm{C} 4 \mathrm{~B}-\mathrm{N} 9 \mathrm{~B}-\mathrm{C} 8 \mathrm{~B}
$$$$
\mathrm{C} 5 \mathrm{~B}-\mathrm{C} 4 \mathrm{~B}-\mathrm{N} 9 \mathrm{~B}-\mathrm{C} 8 \mathrm{~B}
$$$$
\mathrm{N} 3 \mathrm{~B}-\mathrm{C} 4 \mathrm{~B}-\mathrm{N} 9 \mathrm{~B}-\mathrm{C} 10 \mathrm{~B}
$$$$
\mathrm{C} 5 \mathrm{~B}-\mathrm{C} 4 \mathrm{~B}-\mathrm{N} 9 \mathrm{~B}-\mathrm{C} 10 \mathrm{~B}
$$$$
\mathrm{N} 7 \mathrm{~B}-\mathrm{C} 8 \mathrm{~B}-\mathrm{N} 9 \mathrm{~B}-\mathrm{C} 4 \mathrm{~B}
$$$$
\mathrm{N} 7 \mathrm{~B}-\mathrm{C} 8 \mathrm{~B}-\mathrm{N} 9 \mathrm{~B}-\mathrm{C} 10 \mathrm{~B}
$$$$
\mathrm{C} 4 \mathrm{~B}-\mathrm{N} 9 \mathrm{~B}-\mathrm{C} 10 \mathrm{~B}-\mathrm{O} 10 \mathrm{~B}
$$$$
\mathrm{C} 8 \mathrm{~B}-\mathrm{N} 9 \mathrm{~B}-\mathrm{C} 10 \mathrm{~B}-\mathrm{O} 10 \mathrm{~B}
$$$$
\mathrm{C} 4 \mathrm{~B}-\mathrm{N} 9 \mathrm{~B}-\mathrm{C} 10 \mathrm{~B}-\mathrm{O} 11 \mathrm{~B}
$$$$
\mathrm{C} 8 \mathrm{~B}-\mathrm{N} 9 \mathrm{~B}-\mathrm{C} 10 \mathrm{~B}-\mathrm{O} 11 \mathrm{~B}
$$$$
\mathrm{O} 10 \mathrm{~B}-\mathrm{C} 10 \mathrm{~B}-\mathrm{O} 11 \mathrm{~B}-\mathrm{C} 12 \mathrm{~B}
$$$$
\mathrm{N} 9 \mathrm{~B}-\mathrm{C} 10 \mathrm{~B}-\mathrm{O} 11 \mathrm{~B}-\mathrm{C} 12 \mathrm{~B}
$$$$
\mathrm{C} 10 \mathrm{~B}-\mathrm{O} 11 \mathrm{~B}-\mathrm{C} 12 \mathrm{~B}-\mathrm{C} 13 \mathrm{~B}
$$$$
\mathrm{O} 11 \mathrm{~B}-\mathrm{C} 12 \mathrm{~B}-\mathrm{C} 13 \mathrm{~B}-\mathrm{C} 18 \mathrm{~B}
$$$$
\mathrm{O} 11 \mathrm{~B}-\mathrm{C} 12 \mathrm{~B}-\mathrm{C} 13 \mathrm{~B}-\mathrm{C} 14 \mathrm{~B}
$$

$118.0(11)$
$120.47(15)$
$118.1(13)$
$121.4(12)$
$119.34(16)$
$121.1(12)$
$119.6(12)$
$120.34(17)$
$119.3(11)$
$120.4(11)$
$120.23(14)$
$119.0(10)$
$120.8(10)$

$177.65(12)$
$-0.7(2)$
$-177.94(13)$
$0.4(2)$
$177.97(13)$
$-0.8(2)$
$1.4(2)$
$-177.62(11)$
$178.68(13)$
$-0.33(15)$
$1.3(2)$
$-176.89(10)$
$-178.12(14)$
$-1.61(19)$
$0.0(2)$
$176.55(10)$
$176.41(15)$
$-0.18(15)$
$0.65(16)$
$-178.30(14)$
$0.67(14)$
$-7.6(2)$
$171.37(13)$
$-0.87(16)$
$-172.23(12)$
$-173.60(13)$
$-4.4(2)$
$5.50(19)$
$174.71(12)$
$2.0(2)$
$-177.00(11)$
$174.38(11)$
$-6.13(18)$
$173.70(12)$




$\begin{array}{ll}\mathrm{C} 18 \mathrm{~A}-\mathrm{C} 13 \mathrm{~A}-\mathrm{C} 14 \mathrm{~A}-\mathrm{C} 15 \mathrm{~A} & -0.5(2) \\ \mathrm{C} 12 \mathrm{~A}-\mathrm{C} 13 \mathrm{~A}-\mathrm{C} 14 \mathrm{~A}-\mathrm{C} 15 \mathrm{~A} & 179.89(14) \\ \mathrm{C} 13 \mathrm{~A}-\mathrm{C} 14 \mathrm{~A}-\mathrm{C} 15 \mathrm{~A}-\mathrm{C} 16 \mathrm{~A} & 1.2(2) \\ \mathrm{C} 14 \mathrm{~A}-\mathrm{C} 15 \mathrm{~A}-\mathrm{C} 16 \mathrm{~A}-\mathrm{C} 17 \mathrm{~A} & -1.1(2) \\ \mathrm{C} 15 \mathrm{~A}-\mathrm{C} 16 \mathrm{~A}-\mathrm{C} 17 \mathrm{~A}-\mathrm{C} 18 \mathrm{~A} & 0.3(2) \\ \mathrm{C} 14 \mathrm{~A}-\mathrm{C} 13 \mathrm{~A}-\mathrm{C} 18 \mathrm{~A}-\mathrm{C} 17 \mathrm{~A} & -0.3(2) \\ \mathrm{C} 12 \mathrm{~A}-\mathrm{C} 13 \mathrm{~A}-\mathrm{C} 18 \mathrm{~A}-\mathrm{C} 17 \mathrm{~A} & 179.33(14) \\ \mathrm{C} 16 \mathrm{~A}-\mathrm{C} 17 \mathrm{~A}-\mathrm{C} 18 \mathrm{~A}-\mathrm{C} 13 \mathrm{~A} & 0.4(2)\end{array}$

$\begin{array}{ll}\mathrm{C} 18 \mathrm{~B}-\mathrm{C} 13 \mathrm{~B}-\mathrm{C} 14 \mathrm{~B}-\mathrm{C} 15 \mathrm{~B} & 0.0(2) \\ \mathrm{C} 12 \mathrm{~B}-\mathrm{C} 13 \mathrm{~B}-\mathrm{C} 14 \mathrm{~B}-\mathrm{C} 15 \mathrm{~B} & -179.89(14) \\ \mathrm{C} 13 \mathrm{~B}-\mathrm{C} 14 \mathrm{~B}-\mathrm{C} 15 \mathrm{~B}-\mathrm{C} 16 \mathrm{~B} & -0.1(2) \\ \mathrm{C} 14 \mathrm{~B}-\mathrm{C} 15 \mathrm{~B}-\mathrm{C} 16 \mathrm{~B}-\mathrm{C} 17 \mathrm{~B} & 0.0(2) \\ \mathrm{C} 15 \mathrm{~B}-\mathrm{C} 16 \mathrm{~B}-\mathrm{C} 17 \mathrm{~B}-\mathrm{C} 18 \mathrm{~B} & 0.3(2) \\ \mathrm{C} 14 \mathrm{~B}-\mathrm{C} 13 \mathrm{~B}-\mathrm{C} 18 \mathrm{~B}-\mathrm{C} 17 \mathrm{~B} & 0.3(2) \\ \mathrm{C} 12 \mathrm{~B}-\mathrm{C} 13 \mathrm{~B}-\mathrm{C} 18 \mathrm{~B}-\mathrm{C} 17 \mathrm{~B} & -179.87(14) \\ \mathrm{C} 16 \mathrm{~B}-\mathrm{C} 17 \mathrm{~B}-\mathrm{C} 18 \mathrm{~B}-\mathrm{C} 13 \mathrm{~B} & -0.4(2)\end{array}$

Hydrogen-bond geometry $\left(A,{ }^{\circ}\right)$

\begin{tabular}{lllll}
\hline$D-\mathrm{H} \cdots A$ & $D-\mathrm{H}$ & $\mathrm{H} \cdots A$ & $D \cdots A$ & $D-\mathrm{H} \cdots A$ \\
\hline $\mathrm{N} 2 A-\mathrm{H} 2 A \cdots \mathrm{N} 7 B^{\mathrm{i}}$ & $0.890(17)$ & $2.197(17)$ & $3.0771(17)$ & $169.9(14)$ \\
$\mathrm{N} 2 A-\mathrm{H} 2 B \cdots \mathrm{N} 1 B$ & $0.861(17)$ & $2.212(18)$ & $3.0634(16)$ & $169.9(15)$ \\
$\mathrm{N} 2 B-\mathrm{H} 2 D \cdots \mathrm{N} 3 A$ & $0.836(18)$ & $2.302(19)$ & $3.1378(17)$ & $177.8(17)$ \\
$\mathrm{N} 2 B-\mathrm{H} 2 D \cdots \mathrm{O} 10 A$ & $0.836(18)$ & $2.464(17)$ & $2.7501(15)$ & $101.1(13)$ \\
$\mathrm{N} 2 B-\mathrm{H} 2 C \cdots \mathrm{O} 10 A$ & $0.886(18)$ & $2.432(17)$ & $2.7501(15)$ & $101.6(13)$ \\
$\mathrm{C} 8 B-\mathrm{H} 8 B \cdots \mathrm{N} 7 A^{\mathrm{ii}}$ & $0.915(17)$ & $2.375(17)$ & $3.2779(18)$ & $169.1(14)$
\end{tabular}

Symmetry codes: (i) $-x+2, y-1 / 2,-z+1 / 2$; (ii) $x+1, y+1, z$. 